\title{
The circulating TGF-beta contributes to the remyelination in CNS
}

\author{
Machika Hamaguchi, Rieko Muramatsu, Toshihide Yamashita
}

\section{Department of molecular neuroscience, Graduate school of medicine, Osaka University, Japan}

Remyelination is the reconstitution system of myelin sheaths by oligodendrocytes around axons after demyelination. Remyelination requires expression of myelin-constituting proteins in oligodendrocyte and brings restoration of salutatory conduction and facilitation of functional recovery. In this study, we found that the blood component, which leaks into the CNS after demyelination because of blood brain barrier disruption, promotes oligodendrocyte differentiation.

We prepared oligodendrocyte precursor cells (OPCs) from postnatal mice brain and cultured the cells in the presence of serum obtained from adult mice. We found that serum promoted MBP (myelin basic protein)-positive area extension which is one of the major myelin-constituting protein. To identify the molecules in the serum which promotes MBP expression, we performed pharmacological screening. We discovered that the TGF-beta receptor antagonist prevented serum-induced MBP-positive area extension, therefore hypothesized that TGF-beta in the serum facilitates oligodendrocyte differentiation. We treated OPCs with recombinant TGF-beta and observed promotion of MBP-positive area extension. Next, we caused artificial demyelination to the mice and observed TGF-beta increase in the demyelination lesion site assessed by immunohistochemistry. We produced the mice lacking the TGF-beta receptor in oligodendrocytes and the mice lacking TGF-beta in blood which were deleted platelets by intravenously administrated CD41 antibody because platelet is the major source of blood TGF-beta. Then, we caused artificial demyelination to the both mice and found that remyelination restoration were reduced. These dates suggest that the blood TGF-beta leaked into the CNS and promotes myelin-constituting protein expression and remyelination. Furthermore, we found that human oligodendrocyte also expressed TGF-beta receptor and expressions of myelin-constituting proteins in cultured human oligodendrocyte were promoted by TGF-beta stimulation. These dates provides that the TGF-beta might have application possibility to therapy of demyelinating disease. 